\title{
Alcances de una comunidad de aprendizaje en la formación docente ${ }^{1}$
}

\author{
Yenifer Carolina Acosta ${ }^{2}$ \\ Secretaría de Educación Distrital, Bogotá, Colombia \\ caroac25@yahoo.es \\ Diana Marcela Poveda ${ }^{3}$ \\ Secretaría de Educación Distrital, Bogotá, Colombia \\ diana.poveda@ustadistancia.edu.co
}

1 Artículo de investigación, derivado de la investigación realizada para optar el título de Magister en Educación en la Universidad Santo Tomas, Bogotá, Colombia.

2 Licenciada en educación preescolar y primaria de la Corporación Universitaria Iberoamericana. Magister en Educación, Sublínea de investigación: Sociedad de la información y el conocimiento. Universidad Santo Tomás. Docente de básica primaria.

3 Licenciada en educación básica con énfasis en humanidades: español y lenguas extranjeras de la Universidad Pedagógica Nacional (2008). Magister en Educación, Sublínea de investigación: Sociedad de la información y el conocimiento. Universidad Santo Tomás. Docente de inglés 


\section{Alcances de una comunidad de aprendizaje en la formación docente}

\section{Resumen}

Esta investigación analiza los alcances de una comunidad de aprendizaje en la formación docente. La muestra poblacional que intervino en este propósito corresponde a un grupo de siete maestros de inglés de los colegios María Mercedes Carranza I.E.D. y Saludcoop Norte I.E.D., quienes trabajan en el sector público en los niveles de primaria y secundaria. La ruta metodológica de la investigación abordó el paradigma cualitativo, el enfoque hermenéutico y la técnica de etnografía virtual.

Palabras claves: Comunidad de aprendizaje, enseñanza de inglés, TIC y formación docente.

\section{The Scope a community of learning in teacher training}

Abstract

This research analyzes the scope of a community of learning in teacher training. The show from a population study group that intervened in this research corresponds to a group of seven English teachers from María Mercedes Carranza I.E.D. School and Saludcoop North European Institute, who work in the public sector at primary and secondary levels. The methodological research path opted for a qualitative paradigm, the hermeneutic approach and the technique of virtual Ethnography.

Keywords: Learning community; English teaching; ICT; teacher training

\section{Alcances de uma comunidade de aprendizagem na formação docente}

Resumo

Esta pesquisa analisa os alcances de uma comunidade de aprendizagem na formação docente. A amostra de população que faz parte deste objetivo é um grupo de sete professores de inglês das escolas María Mercedes Carranza I.E.D. e Saludcoop Norte I.E.D., que trabalham no setor público na educação primária e secundária. O caminho metodológico da pesquisa, inclui o paradigma qualitativo, o enfoque hermenéutico e a técnica de etnografia virtual.

Palavras chave: comunidade de aprendizagem; ensino do inglês; Tecnologias da Informação e Comunicação; formação docente. 


\section{Introducción}

En la actualidad, la formación que tiene un maestro juega un papel importante a la hora de enseñar y de poner en práctica los conocimientos adquiridos durante el ejercicio de su profesión, por lo tanto, las habilidades que poseen los profesores para comunicar asertivamente aquello que desean enseñar, son sencillamente el reflejo de la formación que han adquirido en la universidad o en sus lugares de trabajo. Los maestros que poseen una formación integral, pueden promover en sus estudiantes la construcción de conexiones entre los conocimientos y la vida cotidiana. Sin embargo, es común basar la enseñanza en los contenidos de un libro sin contemplar las condiciones sociales, políticas y culturales que rodean al aprendiz, lo cual dificulta el desarrollo del pensamiento crítico y no permite que lo aprendido en la escuela supla las necesidades reales de una comunidad.

En el contexto Colombiano, los maestros cuentan con jornadas laborales prolongadas, en las que predominan muchas horas declasey escasos momentos para dialogarentre pares y compartir ideas innovadoras y creativas, que surgen de sus experiencias y de las maneras en que abordan los procesos de enseñanza. Así, se hace evidente la necesidad de generar alternativas, para que la formación de los docentes deje de pensarse en los límites de un aula de clase, y empiece a tomar nuevas formas de debate y discusión pedagógica a través de una comunidad de maestros que tengan intereses y objetivos en común.

Este artículo explora la formación docente, teniendo en cuenta que no sólo es posible crecer profesionalmente a través de la educación impartida por una universidad o instituto, sino que también es importante compartir metodologías, material didáctico y modelos de enseñanza, a través de una comunidad de aprendizaje que supere los límites espacio-temporales, por medio de herramientas tecnológicas como blogs, chats y foros.

La conformación de la comunidad de aprendizaje implicaba la definición de intereses en común entre los maestros participantes, por lo que las investigadoras como facilitadoras y orientadoras de la comunidad consideraron pertinente que el eje de debate fuera la enseñanza de inglés. Esta lengua extranjera, sería el pretexto perfecto para las discusiones, no sólo por la formación de las investigadoras, sino porque el reconocimiento de las formas de hablar, pensar y escribir en inglés es una invitación a romper las fronteras de la lengua materna y aceptar las diferencias entre la cultura inglesa y la latina.

\section{Antecedentes}

Es importante reconocer que ya existen investigaciones que aportan ampliamente a la temática que aborda este trabajo investigativo, y por eso se presenta a continuación 
un análisis de trabajos internacionales y nacionales que dan cuenta de la enseñanza de inglés, las comunidades de aprendizaje y la formación didáctica de maestros como elementos esenciales en los procesos educativos.

Al hacer una búsqueda sobre estudios a nivel internacional relacionados con la enseñanza de inglés, se encontraron dos investigaciones que están relacionadas con el presente proyecto. En primer lugar, se hace referencia a la investigación Venezolana denominada "el impacto de las metodologías de enseñanza utilizadas por el docente sobre la efectividad del aprendizaje del idioma inglés" (Duarte, 2007). En este trabajo se hace evidente que los profesores adolecen de habilidades y destrezas de comunicación en inglés, que permita la enseñanza en las cuatro habilidades (listening, speaking, reading and writing) de manera significativa para los estudiantes. Los maestros involucrados consideran necesario diseñar un material didáctico, sobre metodologías de la enseñanza y el aprendizaje del idioma inglés, dirigido a los niveles de primaria y bachillerato, que contribuya a mejorar la calidad y la pertinencia en la competencia comunicativa de esta lengua extranjera.

Esta exploración sobre las diferentes maneras de enseñar que tienen los maestros de inglés, sirve como base del presente trabajo investigativo, porque describe detalladamente las metodologías y recursos usados por un grupo de profesores de la educación básica y media de cinco colegios del estado de Táchira. Sin embargo, este estudio solo se queda en la descripción de las formas de enseñanza del inglés, y no analiza con profundidad la formación didáctica del profesorado y su impacto en las acciones desarrolladas en clase.

En segundo lugar está la investigación mexicana de la Universidad Pedagógica Nacional (Núñez, 2006), denominada La Enseñanza del inglés en la escuela secundaria. Esta tesis presenta un panorama general de las metodologías y enfoques utilizados en la enseñanza del inglés en secundaria, lo cual es muy valioso para la presente investigación, ya que permite reconocer las diferentes perspectivas que existen para enseñar una lengua extranjera. Además, presenta un individualismo muy marcado entre los profesores y esto es una clara obstrucción para que puedan compartir sus didácticas y metodologías sobre la enseñanza del inglés. Y es precisamente allí, donde surge la necesidad de crear un espacio de reflexión, que conecte a los maestros y les permita construir saberes y actualizarse de forma conjunta.

Siendo la reflexión uno de los elementos vitales en la formación didáctica de los maestros, es preciso abordar las investigaciones sobre comunidades de aprendizaje para tener una mirada global de sus orígenes, su evolución y los alcances que han adquirido en estos últimos tiempos. Las comunidades de aprendizaje son el resultado de las investigaciones que se desarrollan en la entidad CREA, sobre las prácticas educativas y sociales que se fomentan a partir de otras experiencias mundiales. 
El proyecto de comunidades de aprendizaje, empezó a nivel de educación en 1995, en una escuela de educación primaria del país Vasco, desde entonces el número de escuelas que han decidido seguir este modelo inclusivo no ha parado de aumentar, debido a su éxito estas comunidades se han empezado a extender a nivel internacional. El modelo de comunidades de aprendizaje ha demostrado ser efectivo en la superación del fracaso escolar y la mejora de la convivencia multicultural.

A nivel nacional, un estudio representativo de Arguello y Mondragon (2012) maestros en educación de la Universidad Santo Tomás, vincula las comunidades de aprendizaje con el modelo educativo socio-crítico, en el estadio junior del Colegio Jordán de Sajonia. El objetivo de la investigación apunta a interpretar los sentidos y significados que los docentes atribuyen a las experiencias pedagógicas del enfoque comunidades de aprendizaje, en concordancia con el modelo socio-crítico. Los resultados de la investigación hicieron evidente que los docentes descubrieron múltiples sentidos en el ejercicio de la comunidad, el currículo se transformó a través de la práctica y los docentes dieron nuevos significados a términos como pensamiento crítico y refundación de roles de maestro, estudiante y escuela. Este trabajo es importante en la presente investigación, en la medida en que presenta la comunidad de aprendizaje como un recurso que permite dar nuevos significados dentro del proceso de enseñanza, pero se queda en un plano descriptivo que limita la transformación social.

La investigación relacionada con comunidades de aprendizaje en el ámbito educativo se titula: La Comunicación, Interacción en contextos virtuales (Peréz, 2009), esta enmarca la importancia de la comunicación para entender los procesos que se presentan en los ambientes virtuales, ya que los sujetos que intervienen generan vínculos a nivel personal, desarrollando dimensiones cognitivas, afectivas y sociales.

En las últimas décadas se ha venido considerando al alumno como el centro del proceso educativo, y colateralmente se ha descuidado la formación de los docentes, en este entramado las nuevas tecnologías de la información y de la comunicación juegan un papel importante en la educación de este nuevo siglo. Esta nueva educación se centra en la innovación, la globalización, la ruptura de fronteras culturales y lingüísticas y la movilidad virtual de los estudiantes.

La investigación anteriormente citada permite comprender como la formación de los maestros se ha descuidado frente a las nuevas exigencias de la modernidad, esto aporta al presente proyecto porque da relevancia a la formación didáctica docente, reconociendo que el profesor juega un papel importante en los procesos de enseñanza que se llevan a cabo dentro de la escuela.

Al abordar específicamente la formación docente se encuentra un trabajo investigativo de Colombia denominado La formación de profesores de ciencias a 
través de su interacción en comunidades de desarrollo profesional realizado por García (2009) El texto propone formar al profesor como diseñador de proyectos curriculares e investigador de su actuación docente en el aula, y desarrollar actitudes y prácticas docentes más colaborativas, críticas y autónomas.

Este trabajo aporta para la indagación, en la medida en que resalta la necesidad de generar momentos de discusión que evidencien y enriquezcan la formación de los maestros. Además, se presenta claramente la influencia directa que existe entre los procesos reflexivos entre docentes, y el desarrollo de procesos de enseñanza que sean críticos y acordes con la realidad del estudiante. No obstante, la presente investigación se enfoca en la formación didáctica, específicamente de profesores de inglés, y no pretende revisar procesos metacognitivos como concepciones o creencias que existan sobre los procesos de enseñanza.

En contraste, aparece una investigación española denominada "ser y saber ser en la formación didáctica del profesorado" realizada en España por (Contreras, 2010), en ella se hace énfasis en la relación que hay entre la experiencia o formación didáctica que poseen los profesores y su forma de enseñar, relación dada en términos de "experiencia-saber". Este estudio contribuye al trabajo investigativo, ya que nos permite entender que la formación didáctica de los maestros no puede ser mediada sólo en su relación teoría-práctica, o teniendo en cuenta exclusivamente la cantidad de conceptos que se dominen del área que se está enseñando. Una amplia diferencia entre este estudio y la presente investigación, radica en que el autor hace un proceso auto reflexivo de su proceso de formación y experiencias en el aula, sin ampliar los horizontes hacia una formación didáctica que integre a un grupo de colegas.

A partir de este reconocimiento del estado del arte y desde la reflexión sobre la experiencia de enseñar una lengua extranjera en el escenario educativo colombiano, la investigación trazó como objetivo principal, analizar los alcances de una comunidad de aprendizaje en la formación de docentes de inglés, y al mismo tiempo, proyectó algunos propósitos más específicos, tales como el reconocimiento de la comunidad como un espacio para compartir saberes. el uso de diversas estrategias de discusión entre profesores y el esbozo de elementos didácticos valiosos para la enseñanza de inglés, como fruto de los debates dados en la comunidad de aprendizaje. 


\section{Conceptos clave}

\section{Comunidad de aprendizaje}

Las comunidades de aprendizaje nacen de la búsqueda de contacto entre individuos que tienen intereses, ideas y gustos comunes. Para García (2012), las comunidades son comprendidas como: "Agrupaciones de individuos de diferentes niveles que tienen como objetivo fundamental el intentar comprender construcciones sobre la realidad, construcciones que de una u otra manera pueden ser teóricas, construcciones que pueden ser muy vivenciales y construcciones que vienen desde las dos perspectivas"

Estas agrupaciones buscan fundamentalmente generar espacios de diálogo y de construcción colectiva, en donde todos los individuos empiezan a aportar ideas desde su saberes, y prácticas, dando cabida a la construcción del conocimiento, a la interacción y al trabajo colaborativo, los cuales son elementos fundamentales a la hora de trabajar en comunidades. Siendo el término de comunidad de aprendizaje un punto clave en esta investigación, es necesario abordar algunas de sus características:

Tabla 1. Características de las comunidades de aprendizaje

\begin{tabular}{|l|l|}
\hline \multicolumn{1}{|c|}{ Dimensiones } & \multicolumn{1}{c}{ Características } \\
\hline Miembros & $\begin{array}{l}\text { Se asignan o agrupan en función de tareas. } \\
\text { Se conocen entre ellos. } \\
\text { Se realiza una división de las funciones dentro del grupo. }\end{array}$ \\
\hline Tareas u objetivos & $\begin{array}{l}\text { Tema, proyecto o problema definido con un inicio un final claro. } \\
\text { Objetivos de aprendizaje como parte del proyecto. }\end{array}$ \\
\hline Participación & $\begin{array}{l}\text { Grupos pequeños. } \\
\text { Finaliza con la realización de un producto que refleja el } \\
\text { aprendizaje. }\end{array}$ \\
\hline Reproducción & $\begin{array}{l}\text { Transferencia explicita de prácticas, procedimientos y productos. } \\
\text { Lenguaje compartido. }\end{array}$ \\
\hline
\end{tabular}

Fuente: Gros, 2008, p.70

Las comunidades de aprendizaje brindan a los participantes una interacción de forma abierta, ya que su estructura no es de forma piramidal sino circular, en donde todos los aportes de los participes son valiosos para la elaboración de nuevos constructos, que permitan avanzar, elaborar o reelaborar sus estrategias entorno a la enseñanza del idioma extranjero. 


\section{Enseñanza de inglés}

La enseñanza se refiere sencillamente a la acción y efecto de enseñar e incluye procesos como el adoctrinamiento y la instrucción de reglas. A nivel general, aborda las maneras y métodos que se utilizan para instrucción y se caracteriza por seleccionar un conjunto de conocimientos, principios e ideas que son factibles de enseñar a alguien.

Al concebir la enseñanza como la transmisión de conocimientos, se da relevancia a la percepción, principalmente a través de ejercicios de lectura y escritura. Sin embargo, actualmente existen muchas formas de precisar el proceso de enseñanza tales como las exposiciones del docente, el uso de textos, la inclusión de técnicas de participación entre los estudiantes y el aprovechamiento de las nuevas tecnologías.

Si bien es cierto existen diversos procesos de enseñanza, es necesario adaptarlos al área del conocimiento que se quiere enseñar, en el caso de las ciencias exactas es aconsejable utilizar metodologías de resolución de problemas que incluyan procesos de verificación rigurosos, en contraste, las ciencias fácticas requieren de un tratamiento diferente que exalte los procesos naturales de interacción entre los aprendices. De esta manera, el enseñar una lengua extranjera requiere de herramientas propias que potencialicen las habilidades comunicativas (listening, writing, speaking, reading), que permitan llevar a cabo un proceso real de aprendizaje.

Es evidente que el cambio en la manera de concebir la enseñanza, influye directamente en la forma en la que se enseña una lengua extranjera en la escuela, pues la sociedad actual demanda nuevas formas de aproximarse a los saberes, basadas más en la interacción que en los procesos memorísticos aislados, por lo tanto, es posible identificar una ruptura en la concepción de la enseñanza de inglés, pues antes de los años sesenta la estructura era lo más importante, y se daba prelación a aspectos meramente gramaticales y memorísticos.

Sin embargo, en los años siguientes la enseñanza de inglés se inclinó por saber usar la lengua con una intención, propósito y finalidad, apuntando más a una visión comunicativa. Estas nuevas formas de concebir la enseñanza toman como base las teorías lingüísticas y psicológicas, porque son dos disciplinas esenciales para aprender una lengua extranjera, por lo tanto cualquier variación en estos campos del conocimiento, influye directamente en las maneras en que una persona asume la adquisición de la lengua. 


\section{Tecnología de la información y de la comunicación}

Las tecnologías de la información y la comunicación (TIC) han sido un factor vital de cambios a nivel mundial, su crecimiento agigantado y su expansión en los diferentes campos como la política, el componente social y el ámbito educativo, han generado transformaciones y cambios en la actual sociedad. La transformación tecnológica basada en la digitalización de la comunicación permite desarrollar diversas formas de comunicación, nuevos lenguajes, nuevas miradas, nuevas interacciones y nuevos escenarios y formas de acceder y construir conocimiento:

Las redes se convirtieron en la forma organizativa más eficiente como resultado de tres rasgos fundamentales que benefician el nuevo entorno tecnológico: flexibilidad, adaptabilidad y capacidad de supervivencia. Flexibilidad: las redes pueden configurarse en función de los cambios del entorno, manteniendo su objetivo aunque varíen sus componentes. Adaptabilidad: puede expandirse o reducir su tamaño con pocas alteraciones y Capacidad de supervivencia: al no poseer un centro y ser capaces de actuar dentro de una amplia gama de configuraciones (Castell, 2009, pp.49).

Las TIC hacen uso de un lenguaje semiótico integrando un lenguaje oral, escrito, audiovisual, gráfico y a partir de su integración crea un sistema totalmente diferente para la transmisión, acceso y uso de la información. Sistema que atrae y atrapa a niños, niñas, jóvenes y adultos porque ofrece una variada gama de escenarios comunicativos y sociales, en donde se pueden expresar de forma natural, entre ellos se encuentran el correo electrónico, Messenger, chats, blogs y comunidades de aprendizaje, entre otros.

Generar lazos entre las TIC y el entorno educativo es un reto, el cual los maestros deben estar dispuestos a asumir de la mejor forma, ya que es una realidad que atañe a nuestra labor, entender, comprender y vivenciar esa multiplicidad de espacios de interacción requiere que los elementos ya mencionados: adaptabilidad, flexibilidad y conexión comunicativa, contribuyan a una mayor eficiencia y eficacia en las tareas asignadas. De esta manera se hace relevante para este estudio investigativo, según Castell: "La dinámica de las comunidades de aprendizaje en donde la comunicación asincrónica, sincrónica y el trabajo colaborativo conducen a la construcción de conocimiento" (2009).

\section{Formación docente}

Generalmente los individuos buscan mejorar en muchos aspectos a nivel personal, por lo que se aproximan a procesos de formación que enriquezcan la manera en que asumen tareas concretas de la cotidianidad y específicamente en el campo laboral, por lo tanto, es necesario entender la idea de formación a cabalidad: "El concepto de formación corresponde al desarrollo personal que tiene que ver con encontrar 
formas para cumplir con ciertas tareas, para ejercer un trabajo, una profesión" (Ferry, 1977 , pp. 54). Algunas personas consideran que la formación es algo externo, como la implementación de contenidos y programas de aprendizaje, pero realmente el individuo se forma a sí mismo por medio de ciertas mediaciones. Es necesario aclarar aquí, que una mediación puede ser una lectura, una experiencia y hasta el mismo sujeto impartiendo la formación.

En general, existen tres condiciones para que se dé la formación: el lugar, el tiempo y la relación con la realidad. Más allá de ejercer una profesión, para que haya una verdadera formación hay que tomarse un tiempo y un espacio para reflexionar, para volver sobre la labor realizada y tratar de comprender desde diferentes aristas el proceso mismo de la formación.

El centro del proceso formativo es la representación, la cual tienen que ver con la idea de alejarse de la realidad creando imaginarios, juegos e interpretaciones para volver de nuevo sobre ella y observarla desde otra perspectiva. Así, se genera un espacio de transformación, que no corresponde al tiempo y al lugar de la actualidad, sino que permite recrear y representar en la mente el papel del maestro cuando ejerce su labor cotidiana.

Un aspecto esencial de la formación es la motivación, porque quien quiere ser formado o instruido debe manifestar un deseo vehemente de tener el mejor desempeño en el ámbito laboral y en consecuencia accederá de manera fácil y directa a diversos medios de formación. Así lo expresa Ferry "Formarse no es más que un trabajo sobre sí mismo, algo imaginado, deseado e intencionalmente propuesto a través de medios que se ofrecen o que uno procura conseguir" (Ferry, 1977, pp. 43).

\section{Metodología}

Este proyecto es de corte cualitativo y hermenéutico porque busca comprender la formación docente en inglés a través de una comunidad de aprendizaje, proceso en el cual la comunicación juega un papel esencial, ya que los sujetos que intervienen en la investigación se aventuran a compartir sus saberes por medio del diálogo, el consenso y el disenso para demostrar la formación didáctica que poseen a la hora de enseñar.

Para ampliar la visión de la investigación cualitativa, se retoma la postura de los autores Taylor, Bogdan (1986) y Sandín (2003), quienes exponen que un estudio investigativo de carácter cualitativo permite comprender los entornos de forma natural, proporcionando así una mayor interpretación de la experiencia humana. Para Taylor y Bogdan (1986), la investigación cualitativa produce datos descriptivos, las palabras que producen las personas, habladas o escritas y la conducta observable, permitiendo que el investigador desarrolle la habilidad de interpretar los sujetos 
dentro de su entorno natural, apartándose de sus propias creencias, perspectivas y predisposiciones y dándole validez a su investigación.

Por su parte, Sandín propone que la investigación cualitativa atraviesa diversas disciplinas, participa de una gran variedad de discursos o perspectivas teóricas y engloba numerosos métodos y estrategias de recogida de datos. Esta riqueza denota la complejidad y alcance del enfoque cualitativo en el abordaje de la investigación socioeducativa, y requiere que se ensayen clasificaciones o categorías que aporten un orden conceptual en el ámbito de la investigación y permitan la comunicación en la comunidad investigadora (Sandín, 2003, pp. 1).

Teniendo en cuenta estas dos posturas teóricas, es evidente que la naturaleza de esta investigación es cualitativa puesto que dentro de las comunidades de aprendizaje se rescatan los procesos de interacción entre las investigadoras y los sujetos partícipes de la misma, enriqueciendo las prácticas al interpelar y construir saberes. Cabe agregar, que en muchos campos del conocimiento, incluyendo el ámbito educativo, las comunidades utilizan las tecnologías de la comunicación y de la información como herramienta fundamental a la hora de compartir saberes y transversalizar el conocimiento, atendiendo a procesos concretos de flexibilidad, dinamismo, apertura y globalización.

Sumado a esto, es importante concebir la enseñanza de una lengua como algo inacabado, pues esta investigación no aborda un área de carácter científico y finito, sino que hay múltiples voces, diversas formaciones que recrean en el mundo de la enseñanza un imaginario a su vez colectivo y particular. Esa multiplicidad de voces requiere de sujetos "hermeneutas", es decir "interpretadores" de la indiscriminada información que emana de las TIC, exigiendo cada vez más a los sujetos que depuren y tomen una posición crítica frente a la información, todo ello conlleva a interpretar diversos, mensajes, intenciones comunicativas y lenguajes (Gonzálvez, 2009). Así, la investigación realizará una comprensión de la realidad que se vive en el entorno educativo a la hora de enseñar inglés, y en donde las investigadoras precisarán interpretar las múltiples voces puestas en escena a través de una comunidad de aprendizaje.

Este proyecto propone la etnografía virtual como técnica investigativa, ya que la sociedad actual demanda maestros capaces de adaptarse a los cambios en las formas de comunicación y expresión, personas que interactúen en ambientes virtuales y se aventuren a compartir sus experiencias y metodologías de enseñanza con otros compañeros. En esa medida, la comunidad de aprendizaje utiliza el blog de maestros de inglés, para apropiarse y disponer de la virtualidad como un espacio para contribuir a la labor docente. 
Sin embargo, el carácter virtual admite diferentes matices en donde no sólo se pone en juego lo que el informante dice sino las distintas maneras de interpretar que posean las etnógrafas y la relevancia que le den a uno u otro aspecto con el interés de alcanzar los objetivos planteados en la investigación, es decir que, el uso de tecnologías en el ejercicio etnográfico implica un alto grado de flexibilidad interpretativa.

Además, es válido clarificar que la etnografía virtual apunta más al ejercicio de leer y escribir textos, en vez de ocuparse de interacciones directas entre las personas que proporcionan información dentro de la investigación. En ese orden de ideas, la palabra escrita que está latente en el ciberespacio podría equipararse con el análisis de un texto de literatura, en donde las circunstancias que rodean el texto son fundamentales a la hora de interpretar, pues, detrás del ejercicio de escritura hay sujetos con historias de vida, impresiones y posturas que indudablemente reflejan la realidad. Para Hine (2000) algunas características de la etnografía virtual apuntan a:

- Va más allá de lo virtual y aborda la parte social

Está técnica no sólo se ocupa de grupos que comparten a través de medios tecnológicos, sino que trasciende hacia lo social y cultural, por lo que en la comunidad de maestros no sólo se hará una sistematización de la información, sino que se evidenciarán las distintas maneras de enseñar inglés y las particularidades metodológicas de los participantes.

- No se limita a la realidad simulada por los ordenadores

La etnografía virtual se ocupa de los problemas socioculturales utilizando como mediación los artefactos tecnológicos, lo cual se relaciona con los objetivos de esta investigación que buscan revelar la formación de los docentes a través de sus intervenciones en el blog de la comunidad de aprendizaje.

- Puede tratar temas diferentes a los que tratan las comunidades virtuales

El hecho de que esta técnica esté relacionada con la tecnología no implica que trate temáticas exclusivas del entorno virtual, por ejemplo, en esta investigación la enseñanza de inglés es esencial para comprender la formación que tiene un maestro y por ende, dilucidar los recursos con los que puede enriquecer sus prácticas.

En definitiva, la investigación está enmarcada en la técnica de etnografía virtual porque explora las ideas que tienen los maestros sobre la enseñanza de inglés a 
través de encuentros sincrónicos de los participantes, por medio de un foro de manera virtual cuyo propósito es beneficiar las prácticas docentes.

\section{Fases de la investigación}

Aunque en el campo investigativo hay gran variedad de instrumentos, los más acordes para la triangulación de la información fueron: cuestionario de reconocimiento, entrevista y foro virtual, estos permitieron el acercamiento, la caracterización y dinamización de la comunidad de aprendizaje. A continuación se presenta una descripción de los instrumentos teniendo en cuenta cada una de las fases de recolección de datos:

\section{Primera fase: exploración y caracterización}

Se da inicio con un diálogo de saberes con los maestros participes de la investigación, el cual se generó de forma espontánea y amena con la finalidad de hacer una exploración acerca de sus ideas y reflexiones en torno a la enseñanza de un lengua extranjera. Lo anterior permitió el intercambio de algunas anécdotas que han tenido los maestros durante su experiencia en la enseñanza del idioma inglés, y esto a su vez ha incidido en su formación. Por consiguiente, se crea un ambiente de motivación con cada uno de los participantes frente a la comunidad de aprendizaje que se emprendió, llevando a las investigadoras a crear y entregar una invitación como primera aproximación al blog.

La apertura, presentación y socialización de la comunidad de aprendizaje se dio a través del blog denominado http://freeworkshopsforteachers.blogspot.com. Allí se explicó la intención de esta herramienta tecnológica y lo que se pretendía construir en torno a ella, este blog fue ambientado de forma tal, que motivará la participación de los maestros. Las investigadoras nutrieron el blog, con elementos tales como: enlaces, videos, canciones, lecturas, material didáctico con la finalidad de brindar herramientas que aportaran a la práctica docente. Desde el blog se da inicio al cuestionario de reconocimiento.

\section{Cuestionario de reconocimiento}

El cuestionario de reconocimiento fue aplicado en la fase uno, en la que se buscó caracterizar y conocer la población con la que se trabajó. Este instrumento tiene cinco preguntas de tipo cerrado con única respuesta, dirigidas a conocer algunos elementos como: el nivel en el que ejercían la docencia; los años de experticia laboral; herramientas de apoyo que utiliza de tipo físico o tecnológico; y las habilidades que se desarrollan en la enseñanza de inglés. 
FIGURA 1. Cuestionario de reconocimiento

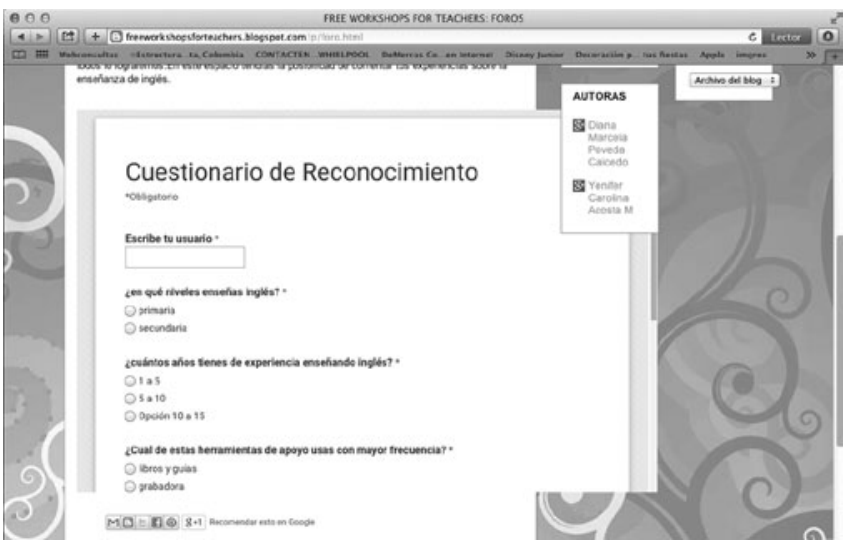

Fuente: blog: http://freeworkshopsforteachers.blogspot.com

\section{Segunda fase: enseñanza de inglés}

\section{Entrevista}

Partiendo del concepto de entrevista el cual es: "una confrontación interpersonal en la cual una persona (el entrevistador) formula a otra (el respondiente) preguntas cuyo fin es conseguir contestaciones relacionadas con el problema de investigación". Kerlinger (1985) citado en (Pérez, 2009, pp. 2). Se dio paso a la aplicación de siete entrevistas semiestructuradas.

FIGURA 2. Cuestionario base de la entrevista

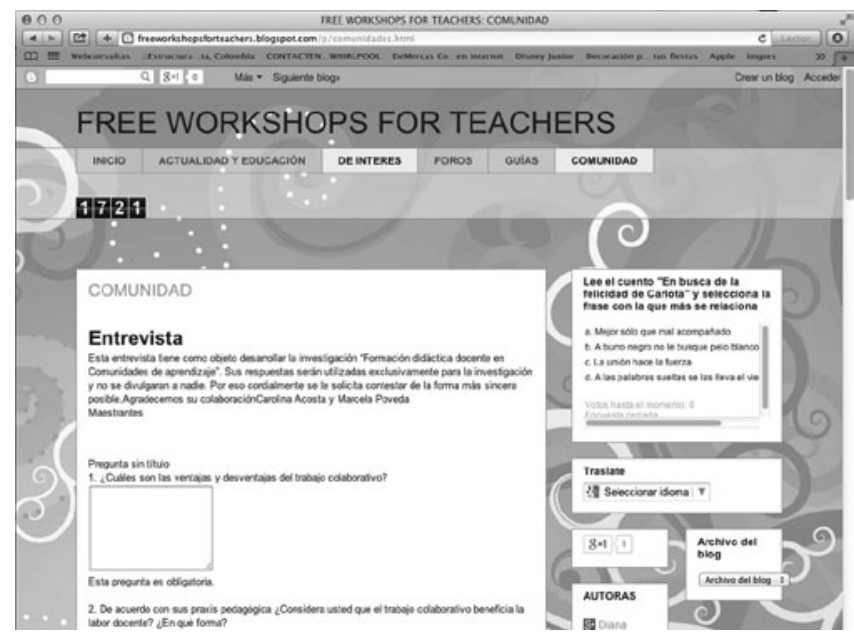

Fuente: blog: http://freeworkshopsforteachers.blogspot.com 
Esta técnica permitió ahondar a través de preguntas los diversos puntos de vista de los maestros de inglés, en esta misma línea, la entrevista fue constituida por 12 preguntas de tipo abierto que fueron elaboradas con relación directa a las categorías y dimensiones claves del proyecto, como se puede observar en la siguiente tabla:

Tabla 2. Relación categoría y dimensión

\begin{tabular}{|c|c|}
\hline Categoría & Dimensión \\
\hline Formación docente & $\begin{array}{c}\text { Flexibilidad } \\
\text { Capacitación }\end{array}$ \\
\hline Comunidad de aprendizaje & $\begin{array}{c}\text { Conexión comunicativa } \\
\text { Trabajo colaborativo }\end{array}$ \\
\hline Enseñanza de inglés & Práctica docente \\
\hline
\end{tabular}

Fuente: Seijas, 2011

Esta entrevista de carácter semi-estructurado se generó con la finalidad de abordar, conocer e identificar elementos que se encuentran latentes en la enseñanza de inglés y que son implícitos en la formación de los maestros. Además, la entrevista fue colgada en el blog para que los docentes ingresaran y respondieran en un límite de tiempo y encontrarán diversos materiales didácticos, los cuales podrían aplicarse en los diferentes niveles en que enseñan.

FIGURA 3. Material didáctico disponible

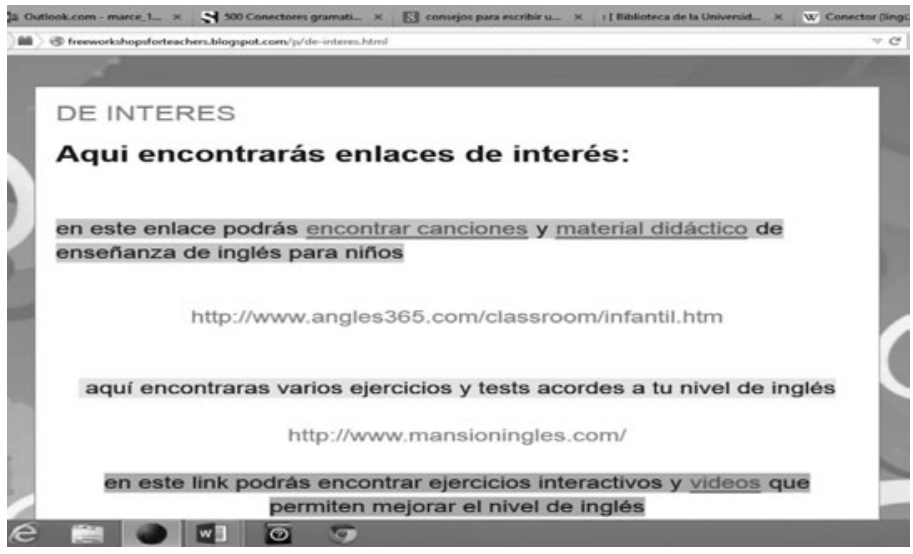

Fuente: blog http://freeworkshopsforteachers.blogspot.com 


\section{Tercera fase: formación y práctica docente}

\section{Foro virtual}

Para Seijas, (2011, p. 1) "El foro es un tipo de reunión donde distintas personas conversan en torno a un tema de interés común. Es esencialmente una técnica de comunicación, realizada en grupos, con base en un contenido de interés general, que origina una discusión". Por consiguiente, se generó el foro virtual como una herramienta de discusión entre los maestros de la comunidad de aprendizaje, para entablar una comunicación cercana a los intereses investigativos. Este foro se colgó en el blog para que los maestros ingresaran y participaran acerca de sus experiencias, tomando como referente su práctica en el aula en la enseñanza del inglés, de igual forma es pertinente aclarar que en el foro se escribieron las indicaciones y el protocolo que debían seguir las participaciones. Así, se utilizó como pretexto un video denominado "El alfarero", el cual buscó generar una relación entre la formación didáctica docente y las formas de enseñanza, partiendo del video se originaron las siguientes preguntas:

1. ¿De qué manera se podría orientar las acciones de los estudiantes para que el aprendizaje sea efectivo?

2. ¿Cuál es ese sello personal que ofreces a los estudiantes mientras se apropian del uso de inglés?

Con la finalidad de orientar a los maestros en la participación en el foro, las investigadoras intervienen a manera de ejemplo, plasmando sus reflexiones e ideas en torno a las preguntas pretexto, marcando así la pauta y la dinámica que debían seguir en el foro.

FIGURA 4. Foro virtual: Compartiendo saberes

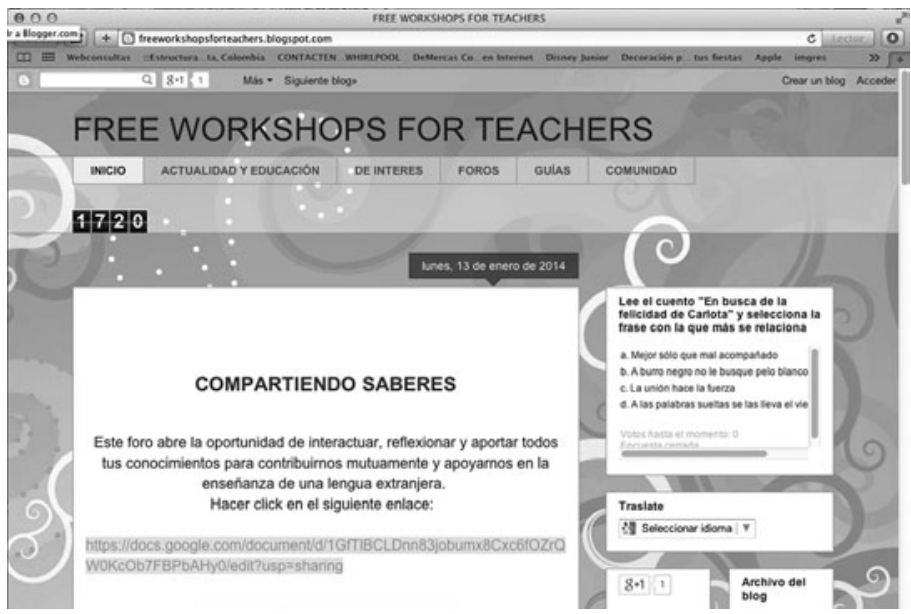

Fuente: blog http://freeworkshopsforteachers.blogspot.com 


\section{Resultados}

\section{Triangulación de los supuestos teóricos con la experiencia investigativa}

Para tener una mayor comprensión de la comunidad de aprendizaje constituida por este ejercicio investigativo, es pertinente triangular la experiencia con los postulados teóricos de Gros y Cabero, autores claves en los horizontes de la investigación. Por su parte Gros (2008) (Tabla 1), retoma elementos de las comunidades de aprendizaje, tales como la determinación de miembros, tareas, objetivos, participación y reproducción, atendiendo a la descripción del trabajo colaborativo. Cabero (2010) habla de tiempos sincrónicos y asincrónicos y de las nuevas formas de comunicación que surgen. En consecuencia, la comunidad conformada en esta investigación aplica esta teoría de la siguiente forma:

a). Los miembros de la comunidad de aprendizaje fueron docentes de inglés de dos colegios públicos, quienes tenían la función de participar en las actividades propuestas a través del blog.

b). El objetivo de la comunidad era brindar un espacio de reflexión e interacción para compartir ideas, experiencias, metodologías y didácticas de la enseñanza de inglés. Este propósito fue dado a conocer de forma clara y puntual en un encuentro presencial entre las investigadoras y los docentes, obteniendo como resultado la sensibilización, motivación y aprobación para formar parte activa de la misma.

c). La participación y los procesos de comunicación son elementos fundamentales a la hora de trabajar en comunidades de aprendizaje, ya que se requiere de un orientador que dinamice y organice las diferentes interacciones entre los miembros, direccionando los aportes, los cuales deben estar encaminados todo el tiempo al objetivo o los objetivos trazados por la comunidad desde un principio.

d). Producto de las múltiples interacciones dadas entre los profesores de inglés se hacen explícitos sus aportes y conocimientos, los cuales han sido construidos desde su experiencia laboral, pedagógica, didáctica entre otras, dándole sentido a la comunidad en la construcción y reelaboración de sus saberes.

e). La participación entre pares académicos evidencia el trabajo colaborativo, ya que todos los participantes de la comunidad de aprendizaje comparten una misma finalidad o meta. en la cual cada uno hace su aporte personal, en busca de un constructo colectivo con miras a enriquecer las maneras que tienen de enseñar inglés.

f). El uso de diversos lenguajes en la comunidad permitió recrear diferentes situaciones, generar ambientes agradables, participativos con una intencionalidad pedagógica definida por las investigadoras líderes del proyecto. 
g). La comunidad ofreció tiempos sincrónicos y asincrónicos a los maestros, para que ellos lograran participar y aportar de forma espontánea y sin estar expuestos a cuestionamientos o discriminación de índole alguna.

\section{Conclusiones}

La experticia pedagógica y tecnológica de los maestros posibilitó que la comunidad de aprendizaje tomará forma y fuerza a medida que los procesos se iban dando, todo esto impulsado por las investigadoras que cumplieron el papel de facilitadoras, motivadoras y guías de la experiencia investigativa, por lo tanto, se da un reconocimiento a la comunidad como espacio, que permitió ofrecer un ambiente novedoso y diferente, en donde los docentes podían expresar sus múltiples conocimientos frente a la enseñanza de inglés.

Desde la comunidad de aprendizaje se logró enunciar diferentes tipos de elementos didácticos usados por los maestros en el aula, algunos elaborados por ellos, fruto de su experiencia laboral y otros instaurados en la web.

Para establecer la comunidad de aprendizaje eje del proyecto, fue de vital importancia resaltar el componente humano, jugar con las actitudes y disponibilidades de tiempo, dar relevancia a la experiencia de los maestros y sus conocimientos sobre el área que se estaba trabajando y hacer hincapié en las interacciones, las cuales se enmarcaron bajo acuerdos de respeto colectivo, académico y profesional.

Una de las ventajas de usar las mediaciones tecnológicas como soporte (el blog en el caso puntual de la investigación), fue que la ubicación en la Red permitió una apertura hacia muchos internautas, para los que el material allí colgado fue atrayente y posiblemente aportó a su vida profesional, laboral y académica.

Las comunidades de aprendizaje transcienden fronteras que superan el terreno de lo físico, y aportan recursos a los maestros que buscan indagar, discutir y reflexionar en torno a la enseñanza de inglés y que se desenvuelven en diversos escenarios educativos.

Las estrategias aplicadas en la comunidad de aprendizaje, permitieron que los maestros expresaran sus ideas sobre la enseñanza de inglés. La entrevista y el foro virtual, fueron pertinentes ya que a través de su implementación permitieron la dinamización e interacción entre los maestros, quienes poco a poco fueron participando con mayor fluidez y tranquilidad. 
Dentro de las estrategias ejecutadas fue importante sortear el elemento del tiempo e intercalar tiempos sincrónicos y asincrónicos, con la finalidad de permitir a los maestros entrar al blog, leer, reflexionar y participar siguiendo ciertos protocolos.

El uso de diversas estrategias (material pedagógico, links, conferencias y videos) persuadió a los maestros, de forma tal, que realizaron procesos de introspección y evaluación sobre su quehacer dentro del aula y la forma como enseñan el idioma extranjero.

La formación docente y la enseñanza de inglés se enriquecieron con metodologías compartidas, tales como dar prelación al tiempo de habla del maestro (Teacher Talking Time- TTT), incluir en las clases la respuesta física total (Total Physical response - TPR) y nutrir el blog http://colorslanguage.blogspot.com/ con recursos didácticos y experiencias educativas.

El aceptar la diferencia y al mismo tiempo reflejarse en el otro, un elemento que enriquece la formación didáctica docente porque en vez de contraponer una metodología de enseñanza con otra, se procuran más procesos de integración y de complementariedad para llevar a cabo el fin último, que es permitir que el estudiante se reconozca como persona inserta en una aldea global y multicultural. Aunque muchos integrantes de la comunidad no estaban familiarizados con las TIC, intentaron comprender y usar los recursos ofrecidos por una sociedad mediática para acceder a las visiones y apuestas pedagógicas del otro.

En definitiva, el compartir saberes permitió reconocer distintas apuestas a la hora de enseñar, pues a veces las metodologías tradicionales apoyan procesos básicos de la escuela como la disciplina y el orden, pero más allá de esto, existen otras maneras de abordar la enseñanza de una lengua extranjera. En las intervenciones que los maestros hicieron en el foro virtual, se dio relevancia al enfoque comunicativo como una forma natural de diálogo en el que afloraron expresiones, costumbres y valores propios de una cultura, es decir, que la comunidad de aprendizaje no sólo sirvió como un espacio de debate entre docentes, sino que podría ser fuente de inspiración para apuestas pedagógicas creativas con los estudiantes. 


\section{Referencias}

(CUCEN), A. N. (6 de Abril de 2011). Lineamientos básicos sobre formación docente de profesores universitarios. Recuperado el 5 de Julio de 2012, de http://www.filo.unt.edu.ar/ mesat/mesa_III_documento_lineamientos.pdf

Acosta, R., Mancina, A., \& Amaya, Y. (2000). Didáctica contemporanéa interactiva para la enseñanza de lenguas. Panamá: Universidad Carlos Manuel Gasteazoro.

Arguello, A., \& Mondragon, U. (2012). Educación crítica y comunidades de aprendizaje. Bogota.

Blanco, R. (2009). Hermeneútica Analogíca y crítica filosófica. México: Torres Asociados.

Cabero, J. (6 de 1 de 2006). Propuestas de Colaboración en Educación a Distancia y Tecnologías para el Aprendizaje. Recuperado el 3 de Junio de 2012, de http://edutec. rediris.es/Revelec2/revelec23/jcabero/cabero.pdf

Cabero, J. (Diciembre de 2010). Edutec. Revista Electronica de Tecnología Educativa. Recuperado el 31 de Mayo de 2012, de http://edutec.rediris.es/Revelec2/revelec34/pdf/ Edutec-e_n34_Cabero_Llorente.pdf

Cabero, J. (Diciembre de 2010). Edutec. Revista Electronica de Tecnología Educativa. 24. Recuperado el 31 de Mayo de 2012, de http://edutec.rediris.es/Revelec2/revelec34/pdf/ Edutec-e_n34_Cabero_Llorente.pdf

Camilloni, A. (2007). El Sabe Didáctico. Buenos Aires: Paidos.

Castell, M. (2009). Comunicación y Poder. Madrid: Alianza.

Contreras, J. (2 de Agosto de 2010). Ser y saber en la formación didáctica del profesorado: una visión personal. Revista interuniversitaria de Formación de Profesorado. 24. Recuperado el 11 de Septiembre de 2012, de http://www.redalyc.org/articulo.oa?id=27419198004

Córdoba, G., \& Araya, K. (septimbre - diciebre de 2010). Principios Epistemológicos y Axiólogicos del saber docente en la enseñanza del inglés. Revista electrónica de actualidades investigativas en Educación. 10. Recuperado el 5 de septiembre de 2012, de http://www. redalyc.org/pdf/447/44717980009.pdf

Duarte, M. (mayo - agosto de 2007). Impacto de las metodologías de enseñanza utilizadas por el docente sobre la efectividad del aprendizaje del idioma inglés. Telos. Recuperado el 16 de Agosto de 2012, de http://www.redalyc.org/pdf/993/99318750008.pdf

Ferry, G. (1977). El trayecto de la Formación los enseñanates entre la teória y la práctica. México: Paidós.

Flecha, G. R. (1999). Las Comunidades de Aprendizaje: Una apuesta por la igualdad. Recuperado el 5 de Agosto de 2012, de http://www.cprceuta.es/comun_aprendizaje/ archivos/flecha_introducc_CCAA.pdf

Gadamer, H. (2006). Estética y Hermeneútica (3a ed). Madrid: Tecnos. 
García Muñoz, T. (Marzo de 2003). El cuestianario como instrumento de invesrigación / evaluación. Recuperado el 25 de Noviembre de 2013, de http://www.univsantana.com/ sociologia/El_Cuestionario.pdf

García, A. (2009). La formación de profesores de ciencias a través de si interacción en comunidades de desarrollo profesional. Recuperado el 12 de Octubre de 2012, de http:// dialnet.unirioja.es/servlet/articulo?codigo $=3782474$

García, A. (4 de Septiembre de 2012). Comunidades de Aprendizaje. (M. Poveda, \& C. Acosta, Entrevistadores) Bogotá.

Goetz, J. P., \& LeCompte, M. D. (1988). Etnografía y diseño cualitativo en investigación educativa. Madrid: Morata.

Gonzálvez, V. (2009). Pedagogía de la autonomía visual. Hacia una Hermeneútica Crítica de la Teconología Comunicactiva. Padado, presente y futuro, 479 -486.

Gros, B. (2008). Aprendizajes, conexiones y artefactos. La producción colaborativa del conocimiento. España: Gedisa.

Gustavo, C., \& Karla, A. (septiembre - diciembre de 2010). Prinicpios Espistemológicos y Axiológicos del saber docente en la enseñanza del inglés. Revista Electrónica Actualudidades Investigativas en Educación, 10(3), 1-41.

Hernan, T., \& Delia, G. (2003). Didáctica General. Coordinación Educativa y Cultural Centro Americana, 9.

Hine, C. (2000). Etnografía virtual. Barcelona: Uoc.

Mattos, L. A. (1974). Compendio de didáctica general. Buenos Aires: Kapelusz.

Medallo, V. (21 de marzo de 2003). Cambio didáctico del profesorado de ciencias experimentales y filosófia de las ciencias. Departamento de didácticas de las ciencias experimentales y de las matemáticas. Universidad Extremadura. Badajoz. Recuperado el 12 de Mayo de 2012, de http://www7.uc.cl/sw_educ/educacion/grecia/plano/html/pdfs/ linea_investigacion/Que_Ciencia_Ensenar_IEC/IEC_043.pdf

Murado, J. (2010). Didáctica de Inglés en Educación Infantil. Métodos de enseñanza para la enseñanza y el aprendizaje de la lengua inglesa (Primera ed.). España: Ideas propias.

Nuñez, J. (Septiembre de 2006). La enseñanza del inglés en la escuela secundaria. Maestría en desrrollo educativo vía medios especialidad en innovavión pedagógica. Recuperado el 13 de Semtiembre de 2012 , de Universidad Pedagógica Nacional Unidad 26 Hermosillo: http://biblioteca.ajusco.upn.mx/pdf/22071.pdf

Peréz, M. d. (Octubre de 2009). La comunicación y la interacción en contextos virtuales de aprendizaje. Recuperado el 8 de Agosto de 2012, de http://www.redalyc.org/articulo. oa?id=68820815003

Salinas, J. (24 al 27 de Noviembre de 2003). Comunidades Virtuales y Aprendizaje digital. EDUTEC'03. 
Sanchéz, A. (Enero de 2009). La sociedad del conocimiento y las TICS: Una inmejorable oportunidad para el cambio docente. Revista de Medios y Educación. 34. Recuperado el 23 de Agosto de 2012, de http://www.sav.us.es/pixelbit/pixelbit/articulos/n34/13.pdf

Sandín, M. P. (2003). Investigación Cualitativa en educación. Fundamentos y tradiciones. Recuperado el 30 de Octubre de 2012, de http://www.postgrado.unesr.edu.ve/acontece/ es/todosnumeros/num09/02_05/capitulo_7_de_sandin.pdf

Santoyo, R. (1981). Algunas reflexiones sobre la coordinación de los grupos de aprendizaje. Perfiles Educativos(118).

Seijas, Y. (1 de Enero de 2011). Manual de usuario para el manejo de un foro virtual. Recuperado el 1 de Diciembre de 2013, de www.iesalc.unesco.org.ve/.../manual_de\%20 usuario_para_\%20manejo_f.

Taylor, S., \& Bogdan, R. (1986). "Introducción: ir hacia la gente" En introducción a los métodos cualitativos de investigación. Recuperado el 25 de Octubre de 2013, de http:// www.geocities.ws/visisto/Biblioteca/TAYLOR

Valls, R., \& Munté, A. (1 de Abril de 2010). Las claves del aprendizaje diálogico en las Comunidades de Aprendizaje. Revista Interuniversitaria de Formación de Profesorado. Recuperado el 6 de Agosto de 2012, de http://www.redalyc.org/articulo. oa?id=27419180001

Vera, M. C. (1987). La didáctica en cuestión. Brasil: Narsea.

Recibido: 27 mayo 2013

Aceptado: 29 julio 2013

Cómo citar:

Acosta, Y.C., Poveda, D.M. (2014) Alcances de una comunidad de aprendizaje en la formación docente. Praxis Pedagógica, 15, 171-192. 